\title{
Polarization Imaging Target Detection Method by Imitating Dragonfly Compound Eye LF-SF Mechanism
}

\author{
Mengxi $\mathrm{Xu}^{1,2}$ \\ ${ }^{1}$ School of Computer Science and Technology, Nanjing \\ Institute of Technology \\ ${ }^{2}$ Nanjing University of Science \& Technology, \\ Nanjing, China \\ mengxi.xu@gmail.com
}

\author{
${ }^{3}$ Xin Wang, ${ }^{3}$ Xijun Yan, ${ }^{3}$ Guofang Lv, ${ }^{1}$ Shengnan \\ Zheng, ${ }^{3}$ Huibin Wang \\ ${ }^{3}$ College of Computer and Information, \\ Hohai University, \\ Nanjing, China
}

\begin{abstract}
Recently, water surface target detection and tracking for sea, lake, or river are challenging research topics. This paper presents a framework of target detection and tracing based on three-channel synchronization polarization imaging and imitation dragonfly compound eye LF-SF (large field- small field) mechanism. This framework can make full use of the advantages of polarization sensitivity of the compound eyes of a dragonfly, and be useful for effective water surface target detection and motion vector estimation.
\end{abstract}

Keywords- target detection and tracking; bionic information processing; polarization imaging

\section{INTRODUCTION}

Water surface target detection and tracking have become research focuses in image processing and computer vision in recent years. For example, in [1], the arctic marine oilspill program (AMOP) remote sensing project was undertaken in order to evaluate the capability of state-of-the-art sensors to detect oil in ice-infested waters. Kim et al. [2] presented a double layered-background removal filter (DL-BRF) for sea target detection problems. However, it still has some challenges for reliable and effective detection and tracking of water surface target.

Firstly, many water surface information acquisition methods are based on visible imaging. However, due to the varying natural and climatic conditions as well as varying optical attributes of the targets, only using light intensity information of the targets for detecting and tracking can not get good results. Therefore, some researchers have attempted to acquire other optical information for water surface target detection. For instance, Taylor [3] presented to use hyperspectral AVIRIS data for oil spill detection and identification. In [4], the degree of polarization was used for ship and oil-spill detection in linear and hybrid/compact dual-pol SAR. In [5], long wave infrared imaging polarimetry was presented to detect water surface objects.

Secondly, besides collection of water surface images, the design of target detectors is also crucial. Ondini et al. [6] described template-matching techniques for automatic detection of multiple, extended, and low contrast targets in infrared maritime scenarios. In [7], the authors used a background subtraction method and a real-time approximation of level-set-based curve evolution to demarcate the outline of moving vessels in the ocean. In [8], a framework for a camera based vessel recognition system was proposed to enhance the functionalities of current Vessel Traffic Services (VTS) systems. Although these methods promote the development of water surface target detection and tracking, they still have these or those shortages.

To solve these problems, this paper presents a framework of target detection and tracking based on three-channel synchronization polarization imaging and imitation dragonfly compound eye LF-SF mechanism. In the first step, it uses polarization imaging to acquire the water surface images. Then, it designs a novel detector by imitating dragonfly compound eye LF-SF (large field-small field) mechanism. This framework will promote the technology of water surface target detection and tracking.

The rest of the paper is organized as follows. The overall design of the framework is presented in Section 2. Section 3 detailedly describes the contents of the framework, such as the three-channel synchronization polarization imaging system, Stokes vectors measurement component, and the target detection and tracking component by imitating dragonfly compound eye LF-SF mechanism. Conclusions are given in Section 4.

\section{OVERALL DESIGN OF THE PRESENTED FRAMEWORK}

The commonly used light attribute of water surface target for detection is intensity. In fact, light attributes, such as polarization, can also provide important information for target detection. Polarization information shows the surface features, shape, and so on of the water targets [9]. Thus, we will utilize polarization imaging for water surface, which is beneficial to water surface target information acquisition.

In addition, most existing water surface target detectors are dependent on background model or prior knowledge of optical information, so the target detection false alarm rate would be raised by clutter background or little prior optical information. In recent years, many biologists study the abilities of water surface target detection and tracking of the hydrophilic insects, such as dragonfly and backswimmer, and have made a progress. These insects possess compound eyes system, which are arranged by multiple small eyes. In 1996, Horvath and Zeil [10] indicated that the compound eyes of a dragonfly have polarization sensitivity, which is the basis for detecting and tracking water surface targets. 
Moreover, the compound eyes system of a dragonfly can acquire large field and small field data at the same time, which also helps it to detect the water surface targets further [11-15]. Inspired by target detection neurons of the dragonfly visual system, a bionic water surface detector is proposed here. It makes full use of the advantages of the compound eyes system of a dragonfly, and can effectively improve the performance of water surface target detection and tracking.

In summary, the whole framework based on polarization imaging and imitation dragonfly compound eye LF-SF (large field- small field) mechanism is designed. The architecture of the proposed framework is shown in Fig. 1. It has three compnents, which are three-channel synchronization polarization imaging system, Stokes vectors measurement module, and the target detection and tracking module by imitating dragonfly compound eye LF-SF mechanism.

\section{THE COMPONENTS OF THE PRESENTED FRAMEWORK}

\section{A. Three-channel Synchronization Polarization Imaging System}

The three-channel synchronization polarization imaging system utilizes three 14 megapixel MT9F002 CMOS image sensors. The frame rate of each sensor is 13fps. And its operating temperature is $-30^{\circ} \mathrm{C}-+70^{\circ} \mathrm{C}$. This type of sensor has such good properties as waterproof, fog-proof and dustproof, which let it be suitable for field conditions. Its pixel size is $1.4 \mathrm{um}$, with power consumption of less than $260 \mathrm{~mW}$.

It is difficult to plate polarizing film in three directions on the single-chip CMOS image sensor. So we design the threechannel synchronization polarization imaging system using three CMOS sensors. They are installed at three equally spaced angle on the visual axis (diameter $=260 \mathrm{~mm}$ ), and the FPGA processing circuit boards are installed in the center vacancy region. The inner surfaces of lenses at $0^{\circ}, 45^{\circ}$ and $90^{\circ}$ directions are mounted by Schneider $\mathrm{B}+\mathrm{W}$ polarizer. The outer surfaces are mounted by B+W081 or B+W484 short band spectral filters. Based on this design, when the height of the imaging probe is $80 \mathrm{~m}$ and the focal length is $40 \mathrm{~mm}$, the spatial resolution is about $2.6 \mathrm{~cm}$.

At last, EP4CGX150 FPGA chip from Altera is selected to develop the system on a programmable chip (SOPC). I2C serial bus and 12bit parallel bus are utilized for the scanning control of the three channel CMOS sensors and the image transmission. Several $34 \times 12$ bit dual-port RAMs and FIFOs are designed for CMOS sensors' parameter read-write and data cache.

\section{B. Stokes Vectors Measurement}

In this component, the three polarized results will be combined to form an image, that is, the polarization image, by measuring the Stokes vectors. In the estimated polarization image, the contrast between the targets and the background is much higher than that in the intensity image. Finally, the polarization image is inputted into the next module.

\section{Target Detection and Tracking by imitating dragonfly compound eye LF-SF mechanism}

The latest research on the detection of surface ships and oil surface indicates that, even using fairly complete optical information acquisition device, the obtained information is still unstable, and contains a lot of noise. In order to complete the final detection task, the system must also rely on the support of the subsequent algorithm.

We propose our two-dimensional target motion detection and motion vector estimation method, which can achieve the water target position match in consecutive frames. The proposed method combines the theory of engineering simulation of LF-SF system in the compound eye visual system, the application of the "Cell Poll" function, and the electrophysiology research result of the charging and discharging mode of the medulla cells.

This component consists of three divisions: compound eyes simulation, water surface information extraction, and target detection and tracking, as shown in Fig.2.

We imitate the imaging mechanism of dragonfly's compound eyes, which are constituted by many small eyes. Such small eye produces an independent image. Then these images are combined together to form an integrated image by biological neural networks. The virtual eye swarm is bundled by five overlapped local windows $(3 \times 3$ or $5 \times 5)$, which build a virtual eye swarm (5 local window size). The small eye swarm imitates the compound eye mechanism to slide all the eyes to get the overlapping polarization sampling of each window.

The imaging system then simulates the large field and the small field channels, which have different spatial and temporal integration characteristics for polarization information. LF and SF channels also have the sensing mechanisms of rapid polarization and slow depolarization characteristics. Moreover, the imaging system simulates P + and P- two groups of shared cell groups of different polarities, which have the mechanisms of diversion enhancement and conflict suppression. This part also uses "Cell Pool" for optimizing and scheduling LF and SF, in order to highlight the texture, edges, and other features of scene characteristics such as rotation mode and the target movement.

Based on the electric physiology research on medulla cells (STMD) and the simulation of scene judgment mechanism by pulse sequence, we design the target motion detector by imitating STMD charge and discharge mode. The proposed method constructs a discriminant function by the form of an exponential function of e to calculate the maximum likelihood of the current pulse sequence, which at last gives the output of the target movement detector.

\section{CONCLUSIONS}

The detection and tracking of water surface target under clutter background have been crucial problems in many fields. However, many methods at present cannot avoid the influences from clutter background. This paper focuses on a novel framework of water surface target detection and tracking based on three-channel synchronization polarization 
imaging and imitation dragonfly compound eye LF-SF (large field- small field) mechanism. This framework is the development of the techniques of water surface target detection and tracking under complex background, and will be widely used in the future.

\section{ACKNOWLEDGMENT}

This work is supported by the Special Fund for Basic Scientific Research of Central Colleges, Hohai University (Grant No. 2011B09314), China Postdoctoral Science Foundation funded project (Grant No. 2012M511185), and Jiangsu Planned Projects for Postdoctoral Research Funds (Grant No. 1102069C), National Natural Science Foundation of China (Grant No. 61263029).

\section{REFERENCES}

[1] R.A. O'Neil, R.A. Neville, V. Thomson. The Arctic Marine Oilspill Program (AMOP) remote sensing study. Environmental Protection Service report series. Canada, 1983.

[2] S. Kim, Y.g Yang, J. Lee. Horizontal small target detection with cooperative background estimation and removal filters. Proceedings of IEEE International Conference on Acoustics, Speech and Signal Processing, 2011, 1761-1764.

[3] S. Taylor. 0.45- to 1.1 -um spectra of Prudhoe crude oil and of beach materials in Prince William Sound, Alaska. Special report, 1992, 92(5): 8-22

[4] R. Shirvany, M. Chabert, J. Y. Tourneret. Ship and Oil-Spill Detection Using the Degree of Polarization in Linear and Hybrid/Compact Dual-Pol SAR. IEEE Journal of Selected Topics in Applied Earth Observations and Remote Sensing, 2012, 5(3): 885892 .
[5] J. S. Harchanko, D. B. Chenault. Water-surface object detection and classification using imaging polarimetry. In Proceedings of SPIE, 2005, 588815, doi: 10.1117/12.623542.

[6] A. Ondini, G. Marsiglia, L. Fortunato, et al. Techniques for detection of multiple, extended, and low contrast targets in infrared maritime scenarios. Optical Engineering, 2006, 45(12): 126401- 126405.

[7] Z. L. Szpak, J. R. Tapamo. Maritime surveillance: Tracking ships inside a dynamic background using a fast level-set. Expert Systems with Applications, 2011, 38(6): 6669-6680.

[8] D. Bloisi, L. Iocchi, M. Fiorini, et al.. Camera based target recognition for maritime awareness. Proceedings of the 15th International Conference on Information Fusion, 2012: 1982-1987.

[9] W. B. Wang, J. H. Ali, R. R. Alfano, et al.. Spectral Polarization Imaging of Human Rectum-Membrane-Prostate Tissues. IEEE Journal of Selected Topics in Quantum Electronics, 2003, 9(2): 288293.

[10] G. Horvath , J. Zeil . Kuwait oil lakes as insect traps. Nature, 1996, 379(7): 303-304.

[11] F. C. Huang, M. Li, A. Y. Shi, et al.. Insert visual system inspired small target detection for multi-spectral remotely sensed images. Journal of Communication, 2011, 32(9): 88- 95.

[12] L. Z. Xu, L. Min, A. Y. Shi, et al.. Feature Detector Model for MultSpectral Remote Sensing Image Inspired by Insert Visual System. Acta Electronica Sinica, 2011, 39(11): 2497-2501.

[13] L. Z. Xu, A. Y. Shi, F. C. Huang, et al.. A perceptual and computational mechnism for bionic compound eye systems with multi-source information fusion. CAAI Transaction on Intelligent Systems, 2008, 3(4): 328-335.

[14] L. Min, L. Z. Xu, A. Y. Shi, et al.. Image reconstruction algorithm inspired by a bionic compound eye system. CAAI Transaction on Intelligent Systems, 2009, 4(2): 180-187.

[15] L. Z. Xu, X. F. Li, S. X.Yang. Intelligent Information Processing and System Optimization. Intelligent Automation and Soft Computing, 2011, 17(7): 829-831

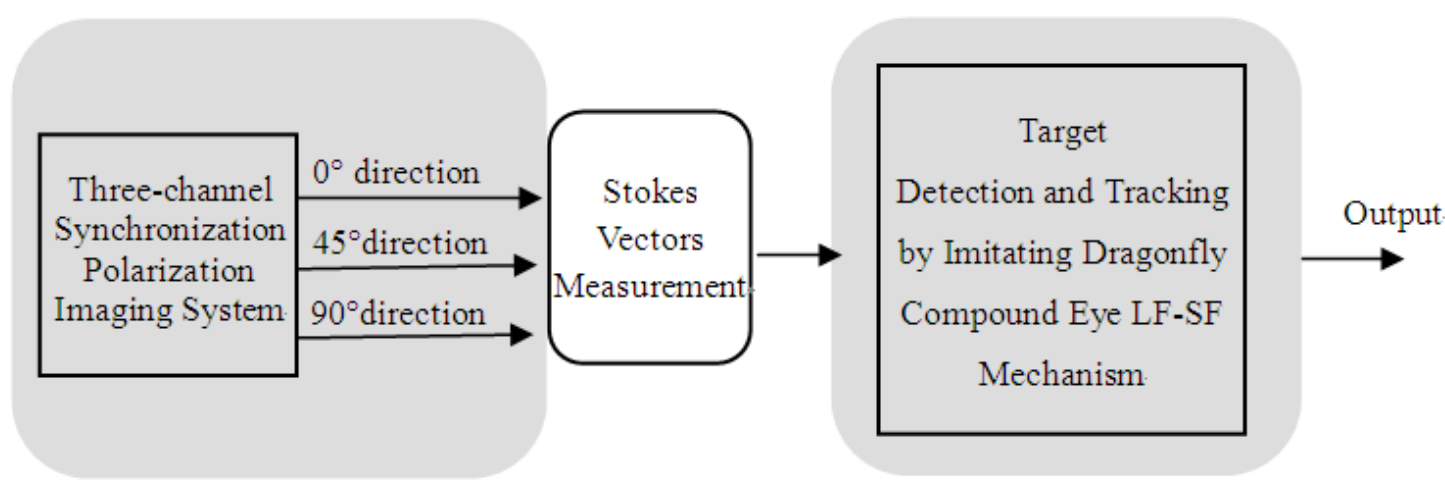

Figure 1. The presented framework based on imitation dragonfly compound eye mechanism

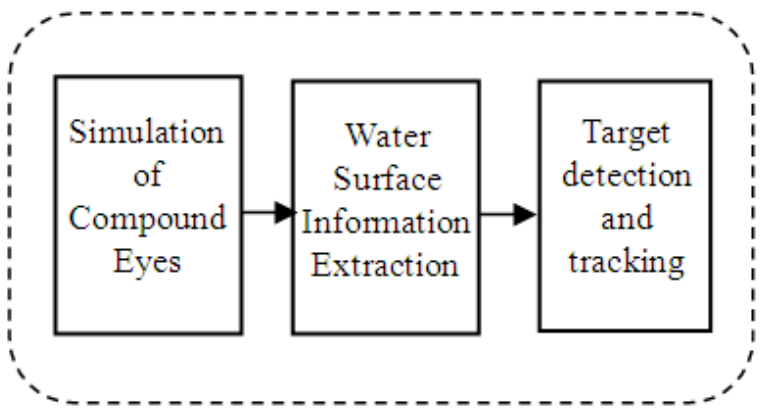

Figure 2. Target detection and tracking by Imitating dragonfly compound eye LF-SF mechanism 